Article

\title{
Frequency Characteristic of Resonant Micro Fluidic Chip for Oil Detection Based on Resistance Parameter
}

\author{
Zilei Yu, Lin Zeng, Hongpeng Zhang *, Guogang Yang, Wenqi Wang and Wanheng Zhang \\ Marine Engineering College, Dalian Maritime University, Dalian 110621, China; \\ ray_yz1417@dlmu.edu.cn or yz1950417@126.com (Z.Y.); bobzl@dlmu.edu.cn (L.Z.); \\ yanggg@dlmu.edu.cn (G.Y.); sarawang@dlmu.edu.cn (W.W.); zwanheng123@dlmu.edu.cn (W.Z.) \\ * Correspondence: zhppeter@dlmu.edu.cn; Tel.: +86-0411-8472-9565
}

Received: 11 May 2018; Accepted: 6 July 2018; Published: 9 July 2018

\begin{abstract}
Monitoring the working condition of hydraulic equipment is significance in industrial fields. The abnormal wear of the hydraulic system can be revealed by detecting the variety and size of micro metal debris in the hydraulic oil. We thus present the design and implementation of a micro detection system of hydraulic oil metal debris based on inductor capacitor (LC) resonant circuit in this paper. By changing the resonant frequency of the micro fluidic chip, we can detect the metal debris of hydraulic oil and analyze the sensitivity of the micro fluidic chip at different resonant frequencies. We then obtained the most suitable resonant frequency. The chip would generate a positive resistance pulse when the iron particles pass through the detection area and the sensitivity of the chip decreased with resonant frequency. The chip would generate a negative resistance pulse when the copper particles pass through the detection area and the sensitivity of the chip increased with resonant frequency. The experimental results show that the change of resonant frequency has a great effect on the copper particles and little on the iron particles. Thus, a relatively big resonant frequency can be selected for chip designing and testing. In practice, we can choose a relatively big resonant frequency in this micro fluidic chip designing. The resonant micro fluidic chip is capable of detecting $20-30 \mu \mathrm{m}$ iron particles and 70-80 $\mu \mathrm{m}$ copper particles at $0.9 \mathrm{MHz}$ resonant frequency.
\end{abstract}

Keywords: resonant frequency; resistance parameter; micro fluidic; oil detection

\section{Introduction}

Hydraulic technology is widely applied in the country's core industries, for example, aerospace, energy, and manufacturing industry due to its advantages of a large transmission force, flexible layout, and high efficiency. In the ship engineering field, hydraulic technology is always applied in major devices such as steering gears, propulsion systems, and lifting equipment. Hydraulic oil as the hydraulic system transmission medium is a kind of high cleanliness fluid, and more than $75 \%$ of hydraulic system failures are caused by hydraulic oil pollution [1]. Thus, predicting and diagnosing the contamination of hydraulic oil is the key to protecting the hydraulic system.

In hydraulic systems, hydraulic oil is inevitably contaminated due to internal generation and external intrusion, among which solid particle contamination is the main source of pollution. Some of these solid particle contaminants are external dust, and metal abrasive particles produced by internal mechanical wear. The latter is the most important cause of mechanical failure in hydraulic systems. The reference data [2-4] shows that the metal abrasive particles in the hydraulic oil have a constant concentration and the size of the abrasive particles is usually 10-20 $\mu \mathrm{m}$ when the hydraulic systems are under normal working conditions. On the contrary, when abnormal wear occurs in the hydraulic systems, the concentration of the metal abrasive particles in the hydraulic oil increases and the size of the particles becomes $50-100 \mu \mathrm{m}$. If the hydraulic system continues to work under this working 
condition, the concentration and size of the metal abrasive particles in the hydraulic oil will still increase until the hydraulic system fails. Thus, how to achieve the detection of particulate contaminants in hydraulic oil is an important content for predicting and diagnosing hydraulic oil contamination.

The main methods currently used for the detection of hydraulic oil include ferrous analysis, spectroscopic analysis, magnetic plug analysis, screen damping, particle counting, etc. [5]. With the exception of the particle counting method, several other methods can only estimate the pollution degree of the oil and require professional laboratory personnel to carry out analysis and measurement. Furthermore, these methods are difficult to achieve in oil on-line monitoring. The particle counting method is a common method for detecting fluid contamination, through the analysis of the amplitude and quantity of the pulses generated by the contaminants passing through the detection area, the size and number of the contaminants can be obtained and the accurate measurement of the oil contaminants can be truly achieved [6,7]. Further, the particle counting method can achieve on-line monitoring, so that it can better predict and diagnose the operation conditions of mechanical equipment and reduce time-consuming and labor-intensive shutdown inspections. Thus, this method has broad application prospects.

Currently, the combination of the micro fluidic electromagnetic technique and particle counting method can achieve some effects, mainly in the three following ways: (1) resistive pulse sensor (RPS), measuring the change of electrolyte resistance when a particle passes through micro channel [8-10], using multiple pores on a single chip as the detection area, detecting the resistance change when particles pass through the area; (2) capacitive counter sensor, measuring the difference in relativity permittivity between oil and metal particles [11,12], using opposite placement metal rods as the electrodes and detecting the changes when particles pass through the electrodes; and (3) inductive counter sensor, measuring the relative different permeability between ferrous particles, nonferrous metal particles, and non-metallic particles [13] using the inductance coil at high frequency condition to detect the particles. However, resistive pulse sensor detection is insensitive due to the fact that oil is non-conductive and non-isothermal. Capacitive counter sensor detection is interfered by a few water droplets in lubricant oil (the relativity permittivity of water and metal particles are both much larger than the relativity permittivity of oil), and it is also incapable of distinguishing the nonferrous metal particles and ferrous particles [14,15]. Du et al. demonstrated the feasibility and proposed a sensor using inductor capacitor (LC) resonant method based on the principle of such an inductive counter sensor and detected $32 \mu \mathrm{m}$ iron particles and $75 \mu \mathrm{m}$ copper particles with different excitation frequency by experiment $[16,17]$. Different from Du detection based on inductance parameter, in this paper, a resonant micro fluidic chip based on electromagnetic theory for detecting resistance parameter is designed and the frequency characteristic of a micro resistance sensor is explored. The theoretical calculations using MATLAB (MATLAB R2015a, The MathWorks, Natick, MA, USA) and experimental verification are performed that the change of resonant frequency has effect on detection. The resonant micro fluidic chip is capable of detecting 20-30 $\mu \mathrm{m}$ iron particles and 70-80 $\mu \mathrm{m}$ copper particles when the resonant frequency is $0.9 \mathrm{MHz}$, which improves the detection accuracy of ferromagnetic particles to some extent

\section{Chip Design and Fabrication}

The micro fluidic resonant oil detection chip is designed to detect the metal debris in oil based on electromagnetic theory as shown in Figure 1. The chip is composited of a plane coil, a chip capacitor, and a micro fluidic channel.

The micro fluidic channel, which is close to the inner wall, goes across the inner hole of the coil, and the coil is connected with the capacitor in parallel. The diameter of micro-channel $D_{1}$ is $300 \mu \mathrm{m}$ (see Figure 2a). The diameter of the coil wire core $D_{2}$ is $70 \mu \mathrm{m}$. There is a coat of insulating paint with a thickness of $10 \mu \mathrm{m}$ around the coil wire core and the diameter of the coil inner hole $D_{3}$ is $900 \mu \mathrm{m}$ (see Figure 2b). 


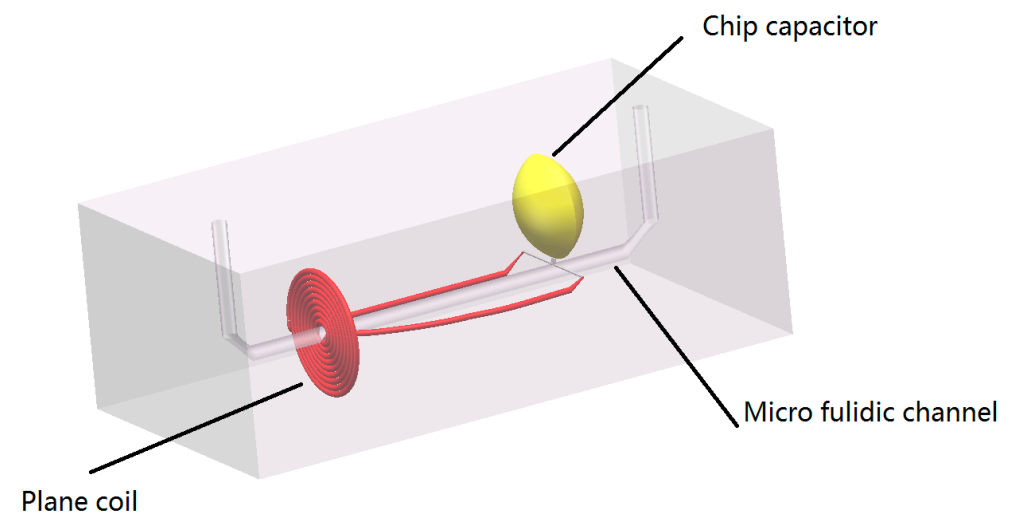

Figure 1. Micro fluidic resonant oil detection chip model figure.

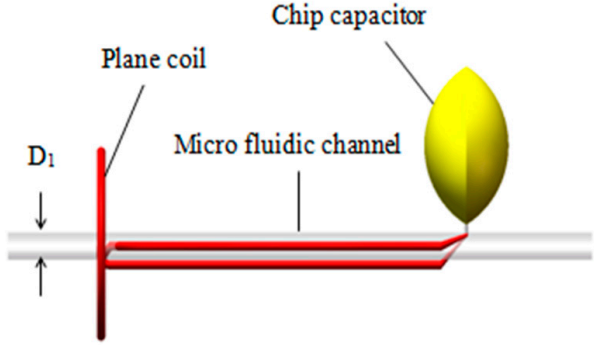

(a)

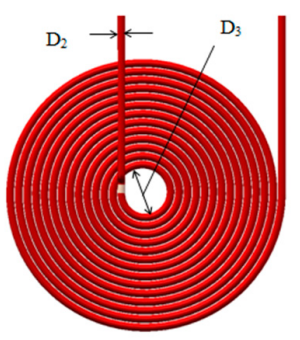

(b)

Figure 2. The design of micro fluidic resonant oil detection chip: (a) cross-section of the chip: $D_{1}$ is the diameter of the micro-channel and (b) a sketch of a single-layer coil: $D_{2}$ is the diameter of the coil wire core and $D_{3}$ is the inner diameter of the single-layer coil.

To fabricate the detection chip, a model of a coil connected with capacitors and a straight micro-channel was made first. The single-layer coil was made of enamel copper wire and wound by a winding machine (SRDZ23-1B, Zhongshan Shili Wire Winder Equipment Co., Ltd., Zhongshan, Guangdong, China); the number of turns is varied according to different experiments. The straight micro-channel model was made of an iron rod with a diameter of $300 \mu \mathrm{m}$ and a length of $7 \mathrm{~cm}$. It was put into the coil inner hole close to the inner wall (see Figure 2a). After that, both the micro-channel model, the capacitor, and the coil were fixed to a glass substrate using glue. The micro-channel model, the capacitor, the coil, and glass substrate formed a chip mold, upon which the liquid polydimethylsiloxane (PDMS) was poured. The chip mold was then placed in a thermostat with a temperature of $80^{\circ} \mathrm{C}$ for $1 \mathrm{~h}$. Finally, after the liquid PDMS was solidified, the straight micro-channel model was removed using pliers and the resonant oil detection chip fabrication was completed. The diameter of the micro-channel model equals to the diameter of the straight micro-channel.

\section{Theoretical Analysis}

The micro fluidic resonant oil detection chip presented in this paper is a resistance sensor, the change of the inductor coil causes the impedance of the entire circuit to change, and the chip detects the real part of the impedance change.

When an excitation voltage is applied to the coil, an alternating current is formed in the coil. Such a coil can be simplified as a circular current-carrying conductor and the magnetic field is shown in Figure 3. It is easy to know that the transverse components of the magnetic field generated by the coils cancel each other out and only have a magnetic field with an axial component. According to the Biot-Savart Law, the center of the coil has the highest magnetic induction. When metal particles pass through this position, the particles are magnetized and the coils generate increased magnetic flux; 
at the same time, particles will also generate eddy current effects at this position which will reduce the magnetic induction of the original magnetic field. For ferromagnetic particles, their relative magnetic permeability is much larger than 1, so when they pass through the detection area, the magnetization field inside them is much larger than the weakened magnetic field generated by the inner eddy current, and the apparent inductance of the coil increase. For non-ferromagnetic metal particles, the relative magnetic permeability is about equal to 1 , so there is no magnetization effect. When the particles pass through the detection area, the magnetization field inside the particle is smaller than the weakened magnetic field generated by the inner eddy current, and the apparent inductance value of the coil reduce.

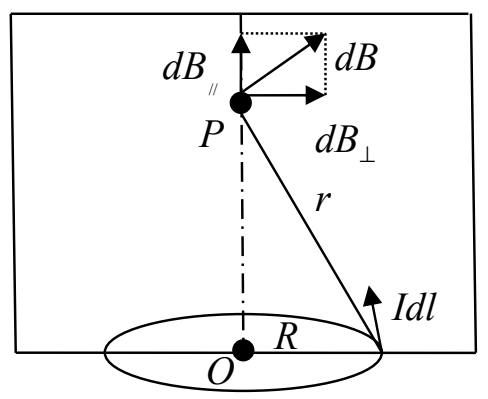

Figure 3. Magnetic field on the circular current-carrying conductor.

\subsection{Particle Differentiate Detection}

The equivalent circuit diagram of the micro fluidic oil detection chip described herein is shown in Figure 4. Among the figure, the inductor coil is equivalent to a pair of series inductance $L_{0}$ and resistor $R_{0}$, the entire circuit is composed of a series branch and a capacitor $C_{0}$ in parallel. The resistance, inductance, and capacitance are expressed by a complex representation, the impedance of the entire circuit is $Z_{0}$. According to the character of the parallel circuit, the branch connected in series with a resistor and an inductor corresponds to a single inductor coil with excitation voltage. Under a constant excitation, a time-harmonic magnetic field is generated and the magnetic induction intensity is constant. When the particles pass through the center of the coil, the particles are magnetized and generate an eddy current effect, resulting in a change in the apparent inductance value of the coil $[18,19]$.

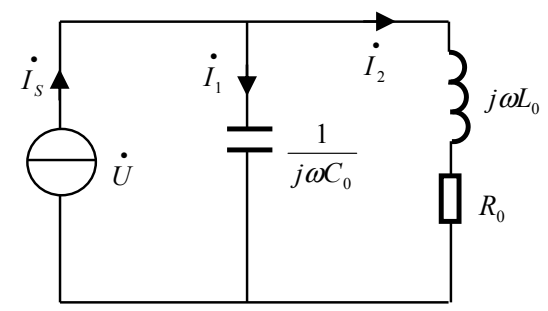

Figure 4. The equivalent circuit of micro fluidic resonant oil detection chip.

When the resonant angular frequency exists in a parallel resonant circuit, it is satisfied by $1-\frac{C_{0} R_{0}}{L_{0}}>0$ and it can be expressed as:

$$
\omega_{0}=\frac{1}{\sqrt{L_{0} C_{0}}} \sqrt{1-\frac{C_{0} R_{0}}{L_{0}}}
$$

When the excitation frequency is the resonant frequency, the entire circuit presents a purely resistive state. When no particles pass through the detection area, the initial equation of the equivalent circuit can be expressed as: 


$$
Z_{0}=\frac{R_{0}+j \omega_{0} L_{0}}{\left(1-\omega_{0}^{2} L_{0} C_{0}\right)+j \omega_{0} R_{0} C_{0}}=\frac{R_{0}+j \omega_{0}\left(L_{0}-\omega_{0}^{2} L_{0}^{2} C_{0}-\omega_{0} R_{0}^{2} C_{0}\right)}{\left(1-\omega_{0}^{2} L_{0} C_{0}\right)^{2}+\omega_{0}^{2} R_{0}^{2} C_{0}^{2}}
$$

The real part of the impedance can be expressed as:

$$
\operatorname{Re}\left(Z_{0}\right)=\frac{R_{0}}{\left(1-\omega_{0}^{2} L_{0} C_{0}\right)^{2}+\omega_{0}^{2} R_{0}^{2} C_{0}^{2}}
$$

When there are metal particles passing through the detection area, the apparent inductance value of the coil changes as described above, so that the changed value produced is the inductance $\Delta L$, and the impedance change value of the entire circuit is $\Delta Z$. At this time, the real part of the changes can be expressed as:

$$
\operatorname{Re}\left(Z_{0}+\Delta Z\right)=\frac{R_{0}}{X_{1}{ }^{2}+X_{2}{ }^{2}}
$$

Among them:

$$
\left\{\begin{array}{l}
X_{1}=\left[1-\omega_{0}^{2}\left(L_{0}+\Delta L\right) C_{0}\right]^{2} \\
X_{2}=\omega_{0}^{2} R_{0}^{2} C_{0}^{2}
\end{array}\right.
$$

Because only the inductance value has changed, only $X_{1}$ has changed in Formula (5). As can be seen, the resonance angular frequency from the previous Formula (1), $X_{1}$ can be expressed as:

$$
X_{1}=\left[1-\frac{1}{L_{0}}\left(1-\frac{C_{0} R_{0}^{2}}{L_{0}}\right)\left(L_{0}+\Delta L\right)\right]^{2}
$$

It can be seen from the beginning that when ferromagnetic particles pass through the detection area, the apparent inductance of the coil increases, that is, $\Delta L>0$; and when the non-ferromagnetic metal particles pass through the detection area, the apparent inductance of the coil decreases, that is, $\Delta L<0$. The Formulas (3) and (6) show that when the ferromagnetic particles pass, $X_{1}$ decreases and the real part of the circuit impedance increases. When non-ferromagnetic metal particles pass, $X_{1}$ increases and the real part of the circuit impedance decreases. Therefore, ferromagnetic particles and non-ferromagnetic particles can be distinguished by detecting the real part of the entire circuit impedance.

\subsection{Effect of Excitation Frequency on Detection}

For a single coil micro fluidic chip, the frequency characteristics have been studied. Through the research, it has found that when the excitation frequency is lower than $2 \mathrm{MHz}$, the frequency change has little effect on the chip detection capability [20]. The inductor coil used in this paper has an inductance of approximately $10 \mu \mathrm{H}$ and a resistance of approximately $0.8 \Omega$. In the previous study, our research team has found that a single coil chip was used for detection the $80-90 \mu \mathrm{m}$ iron particles, the apparent inductance value generated by the inductor coil was changed approximately $4 \times 10^{-5} \mu \mathrm{H}$. And for the 150-160 $\mu \mathrm{m}$ copper particles, the apparent inductance value generated by the inductor coil was changed approximately $-3 \times 10^{-5} \mu \mathrm{H}$. In this paper, the resonant frequency of the detection chip is changed by changing the value of the chip capacitor in parallel, and the frequency of the excitation voltage is set to the resonant frequency. From Formulas (3)-(5), the real part impedance signal amplitude can be expressed as:

$$
\Delta Z=\frac{R_{0}}{\left[1-\omega^{2}\left(L_{0}+\Delta L\right) C\right]^{2}+\omega^{2} R_{0}^{2} C^{2}}-\frac{R_{0}}{\left(1-\omega^{2} L_{0} C\right)^{2}+\omega^{2} R_{0}^{2} C^{2}}
$$

Among them, the capacitor and the excitation angular frequency are variables, the resistance and the inductance of the inductor coil are constants. And the relationship between resonant frequency and resonant angular frequency is:

$$
f=\frac{\omega}{2 \pi}
$$


By MATLAB calculation, we can get the curve of the real part impedance signal amplitude value of the entire circuit as shown in Figure 5 with the excitation frequency $f$ changes.

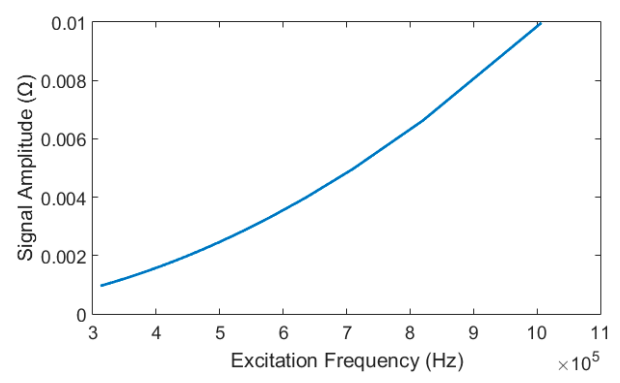

(a)

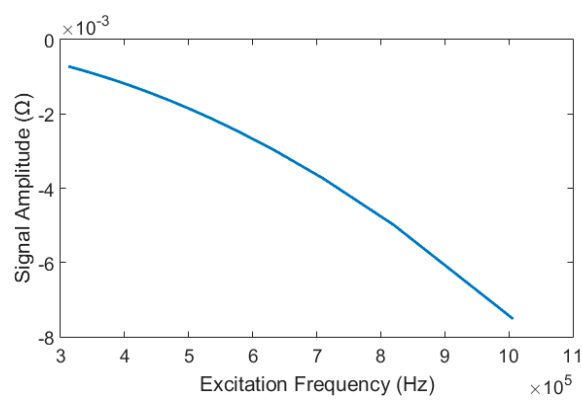

(b)

Figure 5. The relationship between excitation frequency and impedance signal amplitude value: (a) impedance signal value produced by ferromagnetic particle and (b) impedance signal value produced by non-ferromagnetic metal particle.

From the curve in Figure 5, we can see that as the excitation frequency increases, for ferromagnetic particles, the resulting change in the real part of the impedance generated after passing through the detection area increases; for non-ferromagnetic metal particles, the resulting change in the real part of the impedance generated after passing through the detection area decreases. From the foregoing description, it can be seen that the real part of the impedance of the entire circuit increases as the ferromagnetic particles pass through the detection area, and the real part of the impedance of the entire circuit decreases as the non-ferromagnetic particles pass through the detection area. Thence, for the ferromagnetic particles and the non-ferromagnetic particles, the absolute value of the real part of the impedance change produced by the detection area increases with the increase of the excitation frequency.

\section{Experiments and Discussion}

The impedance detection system is shown in Figure 6. It consists of a micro-injection pump (Harvard Apparatus B-85259, Harvard Apparatus, Holliston, MA, USA), a microscope (Nikon AZ100, Nikon, Tokyo, Japan), a micro fluidic chip, an inductance (L), capacitance (C), and resistance (R) meter (Agilent E4980A, Agilent Technologies Inc., Bayan Lepas, Malaysia) and a computer with LabVIEW software (LabVIEW 2011, National Instruments, Austin, TX, USA).

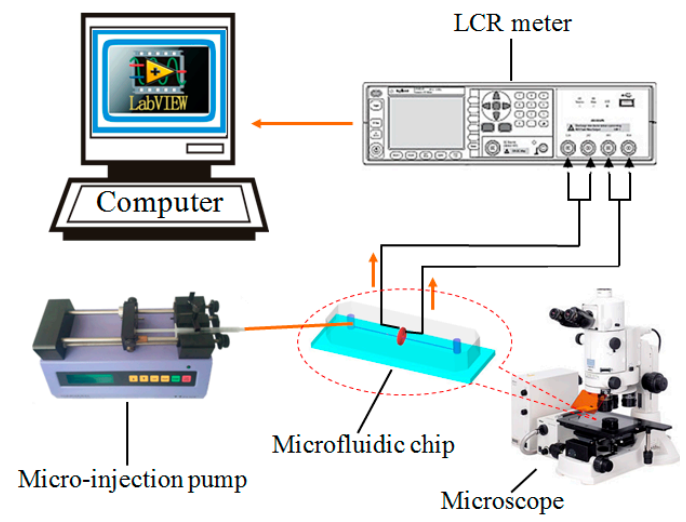

Figure 6. Schematic diagram of impedance detection system. 


\subsection{Experiments Preparations}

In the experiment to investigate the effect of excitation frequency on detection, we used iron particles with sizes of 70-80 $\mu \mathrm{m}$ for ferromagnetic particles, and we used copper particles with sizes of 130-140 $\mu \mathrm{m}$ for non-ferromagnetic particles (Hefei Shatai Mechanical and Electrical Technology Co., Ltd., Hefei, Anhui, China). We weighed $4 \mathrm{mg}$ for each particle using a precision balance (Precisa XS255A, Precisa Gravimetrics AG, Luzern, Switzerland), and mixed the particles with $100 \mathrm{~mL}$ of Marine hydraulic oil (marine hydraulic oil (The Great Wall L-HM 46, Sinopec Lubricant Co., Ltd., Beijing, China) by oscillator (IKA S25, IKA, Staufen, Germany), then put them into plastic test tube as experiment material.

In the experiment of exploring the lowest limit of chip detection, we used iron particles with size of 20-30 $\mu \mathrm{m}, 30-40 \mu \mathrm{m}, 40-50 \mu \mathrm{m}$ for ferromagnetic particles, and we used copper particles with size of 70-80 $\mu \mathrm{m}, 80-90 \mu \mathrm{m}, 90-100 \mu \mathrm{m}$ for non-ferromagnetic particles.

In the experiments, in order to ensure that unrelated variables are consistent when detecting particles, we designed the detection chip as shown in Figure 7. This chip contains 1 inductor and 5 capacitors of different sizes. When the excitation frequency is changed, the coil is connected with different capacitor for detection, and the excitation frequency is keeping unchanged. In this way, the particles can be detected in a chip having the same flow channel and the same inductance coil and the external factors interferes can be relatively reduced.

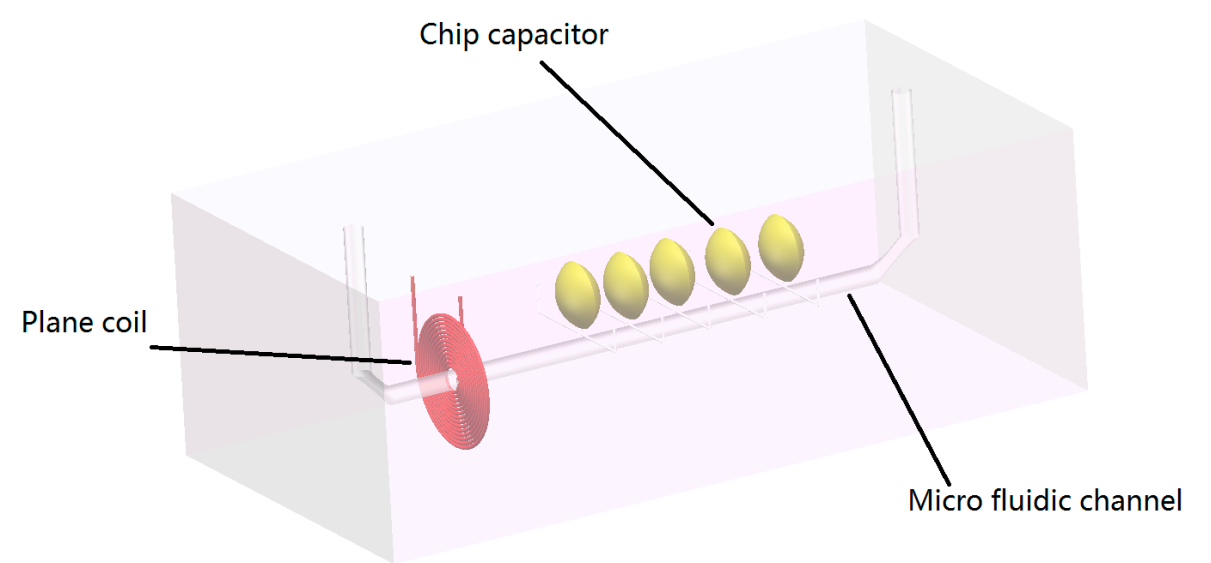

Figure 7. Micro fluidic resonant oil detection chip model figure in the experiments.

\subsection{The Influence of Excitation Frequency on Detection}

In the experiment, we set the excitation voltage of the chip to $2 \mathrm{~V}$ by LCR meter and the injection plastic of the microinjection pump to $40 \mu \mathrm{L} / \mathrm{min}$. Then put 70-80 $\mu \mathrm{m}$ iron particles and 140-150 $\mu \mathrm{m}$ copper particles into the chip and connected micro-injector pump and finally started the experiment.

Among them, the detection results of 70-80 $\mu \mathrm{m}$ iron particles and 140-150 $\mu \mathrm{m}$ copper particles are shown in Figure 8, respectively, at the same voltage and frequency. From Figure 8a,b, we can see that an upward pulse is generated when iron particles pass through the detection area and a downward pulse is generated by copper particles. Thus, we can distinguish the ferromagnetic particles and the non-ferromagnetic particles by the pulse direction judgment. 


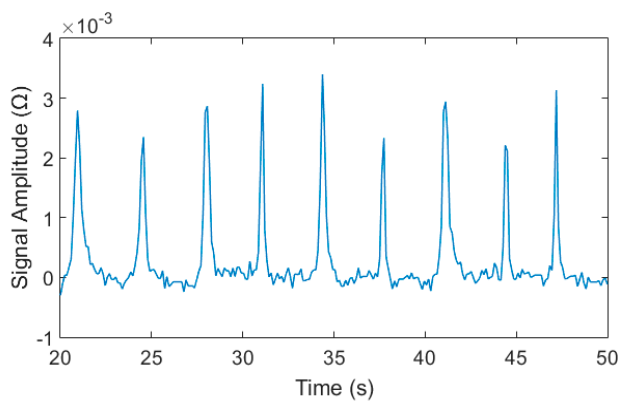

(a)

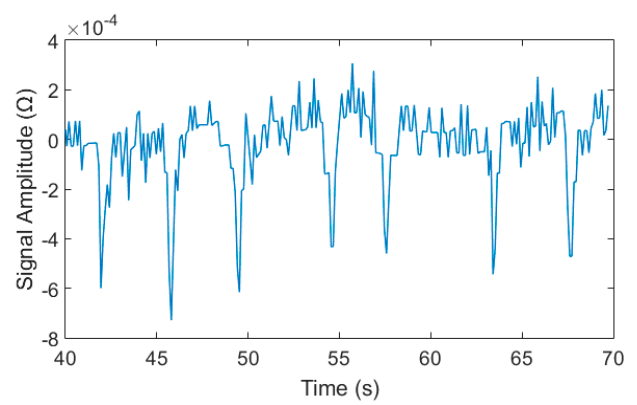

(b)

Figure 8. Detection results of iron and copper particles, the excitation voltage was $2 \mathrm{~V}$ and the excitation frequency was $0.9 \mathrm{MHz}$ : (a) iron particles with sizes ranging from 70-80 $\mu \mathrm{m}$ and (b) copper particles with sizes ranging from $140-150 \mu \mathrm{m}$.

In the experiments, we used 70-80 $\mu \mathrm{m}$ iron particles and $140-150 \mu \mathrm{m}$ copper particles to investigate the influence of excitation frequency on detection precision. The size of the chip capacitors and the excitation frequency are shown in Table 1, the excitation frequency is the resonant frequency corresponding to the connected capacitor. And the highest resonant frequency of the chip is $0.9 \mathrm{MHz}$ in laboratory conditions because the minimum capacitor in our laboratory is $0.010 \mu \mathrm{F}$.

Table 1. Different excitation frequencies in the experiments.

\begin{tabular}{cc}
\hline Chip Capacitors & Excitation Frequency $^{\mathbf{1}}$ \\
\hline $0.10 \mu \mathrm{F}$ & $0.30 \mathrm{MHz}$ \\
$0.068 \mu \mathrm{F}$ & $0.35 \mathrm{MHz}$ \\
$0.047 \mu \mathrm{F}$ & $0.50 \mathrm{MHz}$ \\
$0.022 \mu \mathrm{F}$ & $0.65 \mathrm{MHz}$ \\
$0.010 \mu \mathrm{F}$ & $0.90 \mathrm{MHz}$ \\
\hline
\end{tabular}

${ }^{1}$ Excitation frequency $f=\omega / 2 \pi, \omega$ is excitation angular frequency.

The experimental results are shown in Figure 9a,b, each average data point and its error bar were evaluated by 10 measurements. From the Figure $9 a$, we can see that for the same size of iron particles, with the increase of the excitation frequency, the generated pulse size increase, but the signal-to-noise ratio change negatively with the frequency change. From the Figure $9 b$, we can find that for the same size copper particles, with the increase of the excitation frequency, the generated pulse size increase, and the signal-to-noise ratio (SNR) also change in proportion to the frequency. Furthermore, the amplitude curve of experiments is consistent with theoretical calculations in Figure 5.

\subsection{Detection Limit of the Detection Chip}

From Figure 9 we can also know that the SNR of copper particle detection signal is more effective than the SNR of iron particle detection signal with excitation frequency change. Thus, we have chosen $0.9 \mathrm{MHz}$ as the excitation frequency to investigate the detection lowest limit of the detection chip in the experiments. Moreover, we used iron particles with size of $20-30 \mu \mathrm{m}, 30-40 \mu \mathrm{m}$, and $40-50 \mu \mathrm{m}$ and copper particles with size of 70-80 $\mu \mathrm{m}, 80-90 \mu \mathrm{m}$, and $90-100 \mu \mathrm{m}$ as the experiment's materials.

Through the experiments, we found that the limitation of detection chip is iron particle 20-30 $\mu \mathrm{m}$ and copper particle $70-80 \mu \mathrm{m}$ when the excitation voltage is $2 \mathrm{~V}$, the excitation frequency is $0.9 \mathrm{MHz}$ and the injection flow rate is $40 \mu \mathrm{L} / \mathrm{min}$. The detection signal and the particle figure as shown in Figure 10. 


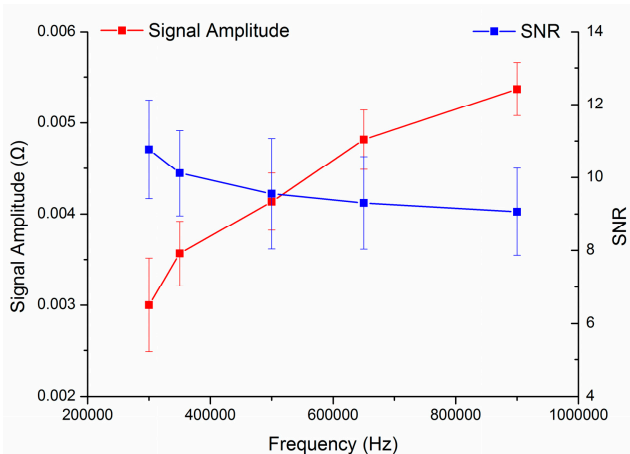

(a)

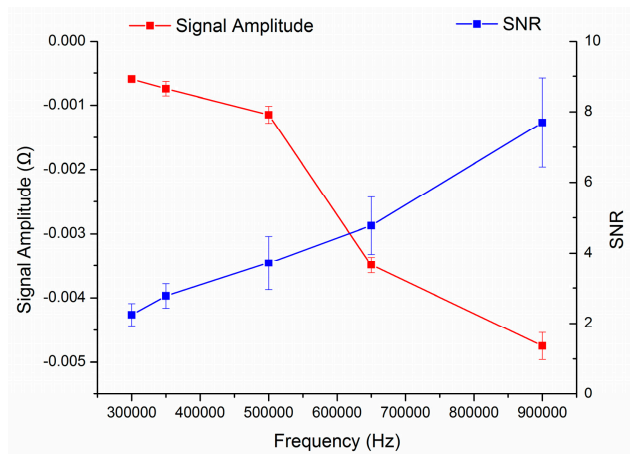

(b)

Figure 9. Influence of particle amplitude and signal-to-noise ratio (SNR) with excitation frequency: (a) 70-80 $\mu \mathrm{m}$ Iron particles detection results, the blue line was SNR, and the red line was signal amplitude and (b) 140-150 $\mu \mathrm{m}$ copper particles detection results, the blue line was SNR, and the red line was signal amplitude.

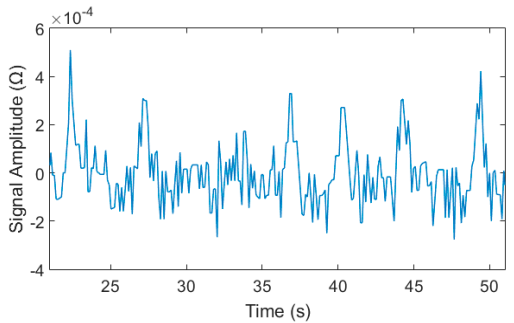

(a)

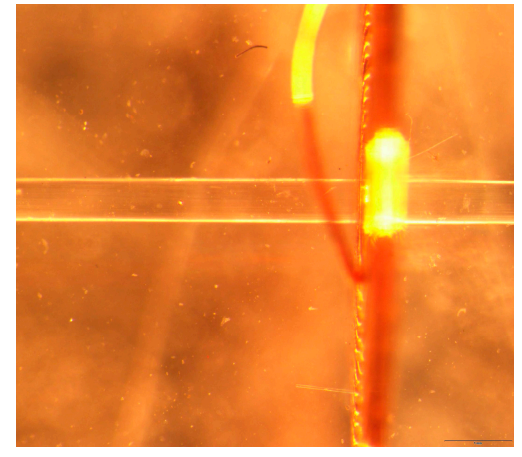

(c)

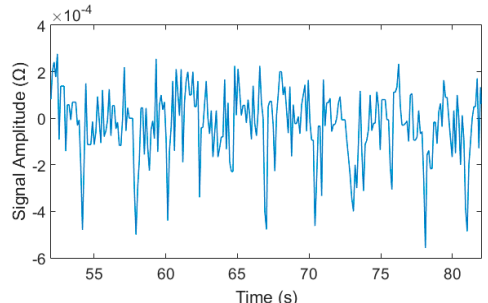

(b)

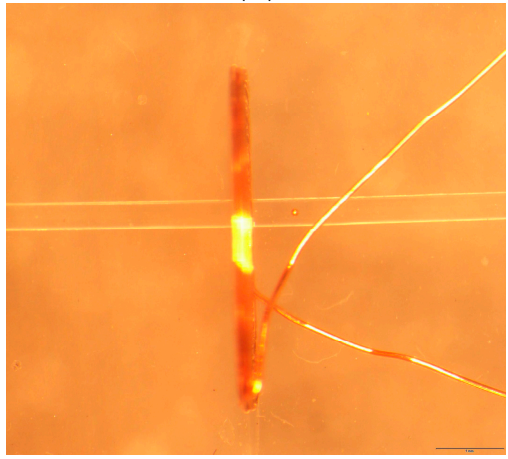

(d)

Figure 10. The limitation detection results of iron and copper particles, the excitation voltage was $2 \mathrm{~V}$ and the excitation frequency was $0.9 \mathrm{MHz}$ : (a) iron particles with sizes ranging from 20-30 $\mu \mathrm{m}$; (b) copper particles with sizes ranging from 70-80 $\mu \mathrm{m}$; (c) 20-30 $\mu \mathrm{m}$ iron particle under microscope; and (d) 70-80 $\mu \mathrm{m}$ copper particle under microscope.

\subsection{Mixtures Detection of the Detection Chip}

The mixtures of 70-80 $\mu \mathrm{m}$ iron particles and $140-150 \mu \mathrm{m}$ copper particles and the mixtures of 20-30 $\mu \mathrm{m}$ and 70-80 $\mu \mathrm{m}$ iron particles were detected at $0.9 \mathrm{MHz}$ and $2 \mathrm{~V}$ condition in experiments to verify that the detection chip can distinguish different size ferromagnetic and non-ferromagnetic particles. And the detection results were shown in Figure 11. The positive signals were generated by iron particles and the negative signals were generated by copper particles in the Figure 10a. The larger positive signals were generated by $70-80 \mu \mathrm{m}$ iron particles and the smaller were generated by $20-30 \mu \mathrm{m}$ particles in the Figure 10b. 


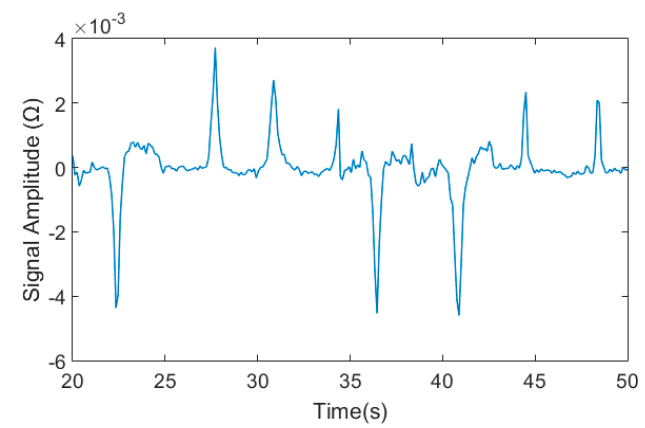

(a)

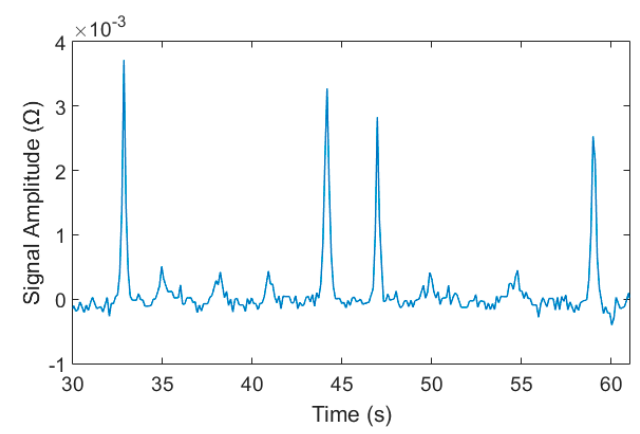

(b)

Figure 11. The mixtures detection result of the chip: (a) the mixtures of 70-80 $\mu \mathrm{m}$ iron particles and 140-150 $\mu \mathrm{m}$ copper particles results, the positive signals were iron particles, the negative signals were copper particles and (b) the mixtures of 20-30 $\mu \mathrm{m}$ and 70-80 $\mu \mathrm{m}$ iron particles detection results, the larger signals were $70-80 \mu \mathrm{m}$ iron particles, the smaller signals were $20-30 \mu \mathrm{m}$ iron particles.

\section{Conclusions}

A resistance micro fluidic detection chip based on resonant circuit for detecting metal particles in hydraulic oil has been designed in this paper. The experimental results are consistent with the theoretical calculations. The chip would generate a positive resistance pulse when the iron particles pass through the detection area, and the chip would generate a negative resistance pulse when the copper particles pass through the detection area, and the detection signal amplitude increased with resonant frequency. With excitation frequency increased, the signal-to-noise ratio decreased for ferromagnetic particles and increased for non-ferromagnetic particles. Since the highest resonant frequency of the chip is $0.9 \mathrm{MHz}$ in laboratory conditions, we used it as the chip's excitation frequency to investigate the limitation of detection and detected 20-30 $\mu \mathrm{m}$ iron particles and 70-80 $\mu \mathrm{m}$ copper particles. We can effectively predict the hydraulic system fault by the size of the contaminants and its location to some extent by distinguishing the contaminants. The condition monitoring and fault diagnosis is significance to the ship's hydraulic system.

The detection accuracy and the throughput of this chip are still limited. In our future works, we will improve the detection accuracy by increase the turns of the coil and improve the throughput by increase the diameter of the fluid channel.

Author Contributions: Z.Y. and L.Z. conceived and designed the experiments; Z.Y. performed the experiments and wrote the first draft of this manuscript; H.Z. and G.Y. aided in theoretical; W.W. and W.Z. aided in harvest, finished writing, and editing of the manuscript.

Funding: This work was supported by the Natural Science Foundation of China (51679022), the Fundamental Research Funds for the Central Universities (3132017013).

Conflicts of Interest: The authors declare no conflict of interest.

\section{References}

1. Zhang, X.; Zhang, H.; Sun, Y.; Chen, H.; Guo, L. Effects of eddy current within particles on the 3D solenoid microfluidic detection chip. Appl. Mech. Mater. 2013, 385, 546-549. [CrossRef]

2. Tucker, J.E.; Schultz, A.; Lu, C.; Sebok, T.; Holloway, C.; Tankersley, L.L.; Reintjes, J. Lasernet fines optical wear debris monitor. In Proceedings of the International Conference on Condition Monitoring, Swansea, Wales, 12-15 April 1999; pp. 445-452.

3. Zhang, H.; Huang, W.; Zhang, Y.; Shen, Y.; Li, D. Design of the microfluidic chip of oil detection. Appl. Mech. Mater. 2012, 117, 517-520. [CrossRef]

4. Du, L.; Zhu, X.; Han, Y.; Zhe, J. High throughput wear debris detection in lubricants using a resonance frequency division multiplexed sensor. Tribol. Lett. 2013, 51, 453-460. [CrossRef] 
5. Du, L.; Zhe, J. On-line wear debris detection in lubricating oil for condition based health monitoring of rotary machinery. Electr. Eng. 2011, 4, 1-9. [CrossRef]

6. Kumar, M.; Mukherjee, P.S.; Misra, N.M. Advancement and current status of wear debris analysis for machine condition monitoring: A review. Ind. Lubr. Tribol. 2013, 65, 3-11. [CrossRef]

7. Wu, T.; Peng, Y.; Wu, H.; Zhang, X.; Wang, J. Full-life dynamic identification of wear state based on on-line wear debris image features. Mech. Syst. Signal Process. 2014, 42, 404-414. [CrossRef]

8. Deblois, R.W.; Bean, C.P. Counting and sizing of submicron particles by the resistive pulse technique. Rev. Sci. Instrum. 1970, 41, 909-916. [CrossRef]

9. Carbonaro, A.; Sohn, L.L. A resistive-pulse sensor chip for multianalyte immunoassays. Lab Chip 2005, 5, 1155-1160. [CrossRef] [PubMed]

10. Wu, X.; Kang, Y.; Wang, Y.; Xu, D.; Li, D.; Li, D. Microfluidic differential resistive pulse sensors. Electrophoresis 2008, 29, 2754-2759. [CrossRef] [PubMed]

11. Sohn, L.L.; Saleh, O.A.; Facer, G.R.; Beavis, A.J.; Allan, R.S.; Notterman, D.A. Capacitance cytometry: Measuring biological cells one by one. Proc. Natl. Acad. Sci. USA 2000, 97, 10786-10790. [CrossRef] [PubMed]

12. Murali, S.; Jagtiani, A.V.; Xia, X.; Carletta, J.; Zhe, J. A microfluidic coulter counting device for metal wear detection in lubrication oil. Rev. Sci. Instrum. 2009, 80, 016105. [CrossRef] [PubMed]

13. Du, L.; Zhe, J.; Carletta, J.; Veillette, R.; Choy, F. Real-time monitoring of wear debris in lubrication oil using a microfluidic inductive coulter counting device. Microfluid. Nanofluid. 2010, 9, 1241-1245. [CrossRef]

14. Flanagan, I.M.; Jordan, J.R.; Whittington, H.W. An inductive method for estimating the composition and size of metal particles. Meas. Sci. Technol. 1990, 1, 381. [CrossRef]

15. Hong, W.; Wang, S.; Tomovic, M.; Han, L.; Shi, J. Radial inductive debris detection sensor and performance analysis. Meas. Sci. Technol. 2013, 24, 125103. [CrossRef]

16. Du, L.; Zhe, J. A high throughput inductive pulse sensor for online oil debris monitoring. Tribol. Int. 2011, 44, 175-179. [CrossRef]

17. Du, L.; Zhu, X.; Han, Y.; Zhan, L.; Zhe, J. Improving sensitivity of an inductive pulse sensor for detection of metallic wear debris in lubricants using parallel LC resonance method. Meas. Sci. Technol. 2013, 24, 075106. [CrossRef]

18. Zhang, X.; Zhang, H.; Sun, Y.; Chen, H.; Zhang, Y. Research on the output characteristics of microfluidic inductive sensor. J. Nanomater. 2014, 2014, 725246. [CrossRef]

19. Yu, Z.; Zhang, H.; Zeng, L.; Teng, H. Detection of metal particles based on micro-fluidic resonant chip. J. Electron. Meas. Instrum. 2017, 31, 1627-1632.

20. Zhang, X. Study on Metal Particle Magnetization in Harmonic Field. Ph.D. Thesis, Dalian Maritime University, Dalian, China, 2014. 